\title{
Análise da Intersetorialidade no Programa Saúde na Escola
}

\author{
Cross-sectorial Analysis in the Health at \\ Schools Program
}

\author{
Isabelle Carolline Veríssimo de Farias ${ }^{I}$ \\ Ronice Maria Pereira Franco de Sál \\ Nilcema Figueiredo \\ Abel Menezes Filho
}

\section{PALAVRAS-CHAVE}

- Saúde Escolar;

- Educação Médica;

- Promoção da Saúde;

- Ação Intersetorial.

\section{KEYWORDS}

- School Health;

- Medical Education;

- Health Promotion;

- Intersectoral Action.

Recebido em: 11/10/2014

Reencaminhado em: 04/09/2015

Aprovado em: 18/01/2016

\section{RESUMO}

A pesquisa objetivou conhecer e analisar o processo da intersetorialidade no Programa Saúde na Escola (PSE)em um município da região metropolitana de Pernambuco. Trata-se de uma pesquisa qualitativa que abordou, por meio de entrevista semiestruturada, profissionais da saúde e da educaçãonas 20 primeiras escolas que aderiram ao PSE desde seu lançamento no município. Os dados foram analisados por meio da análise de conteúdo proposta por Bardin, associada ao programa computacional NVivo®, assim como também foi utilizado o diário de campo. A pesquisa revelou que o maior dilema é operar uma politica necessariamente intersetorial, explicitada num ambiente com atores que possuem agendas setoriais previamente montadas e sem espaço suficiente para a correta execução da política, o que leva à dificuldade de conciliar os tempos institucionais dos vários setores e o comprometimento e envolvimento setorial, impedindo a sustentabilidade das ações. Mudanças estão sendo implementadas, mas o que se observa é a escassez de capacitação e educação permanente, além da inexistência de protocolos que norteiem o desenvolvimento de ações intersetoriais.

\section{ABSTRACT}

This research aimed to identify and analyze the process of cross-sectorialcooperation in the Health at Schools Program in a cityin Pernambuco state. Through semi-structured interviews this qualitative research addressed health care and education professionals in the first 20 schools that joined the PSE since its launch in the city. Data were analyzed using content analysis proposed by Bardin, associated with the computer program NVivo ${ }^{\circledR}$, as well as field diary also being used. The survey revealed that the biggest dilemma is to operate a necessarily cross-sectorial policy explained in an environment with actors that have previously established sectorial agendas and without sufficient space for the correct implementation of the policy, which leads to difficulty in reconciling institutional times of the various sectors and the sector involvement and commitment, preventing the sustainability of actions. Changes are being implemented, but what can be seen is the lack of training and continuing education, besides the lack of protocols to guide the development of cross-sectorial actions. 


\section{INTRODUÇÃO}

As políticas de saúde no contexto brasileiro têm se desenvolvido no reconhecimento da intersetorialidade. Em nenhum outro momento histórico, falou-se tanto em saúde e promoção da saúde como no contexto atual, o que corrobora a valorização do papel de promoção da saúde vinculada ao ambiente escolar como elemento transformador da realidade. Assim, reconhece-se a necessidade de atuação não somente do setor saúde, mas também como o resultado de ações intersetoriais e multidisciplinares, tornando a intersetorialidade uma condição para a prática da Promoção da Saúde ${ }^{1}$.

A intersetorialidade se fundamentanuma prática integradora de ações de distintos setores, que se articulam, complementam e interagem para uma aproximação mais intrincada dos problemas e seu enfrentamento, compartilhando recursos e metas, e desenvolvendo estratégias conjuntamente ${ }^{2}$.

Não se trata apenas de uma estratégia política de intersetorialidade na saúde. É a convicção de que os ganhos positivos em indicadores de saúde requerem um amplo processo de inclusão para a compreensão e transformação da realidade, significando um esforço na interação de diferentes saberes e atores sociais para o estabelecimento de relações horizontais e para a superação de problemas de saúde ${ }^{3}$. Os resultados de saúde alcançados por meio da intersetorialidade são mais efetivos do que o setor saúde alcançaria por si só ${ }^{4}$.

Um dos maiores desafios da Saúde Pública é a utilização das evidências obtidas por meio das diversas fontes de dados (primárias, secundárias, artigos, midiáticas) para aprimorar, reorganizar e transformar as práticas rotineiras. Nesse contexto, os estudos que apresentam evidências têm sido um assunto de interesse crescente de governos e diferentes atores envolvidos na implementação de projetos e de ações em promoção da saúde ${ }^{5}$.

Instituído pelo Decreto Presidencial no 6.286, de 5 de dezembro de 2007, o Programa Saúde na Escola (PSE) resulta de uma parceria entre os ministérios da Saúde e da Educação. Seu objetivo principal é contribuir para a formação integral dos estudantes da rede pública de educação básica por meio das ações de prevenção, promoção e atenção à saúde, integrando as redes do Sistema de Educação e do Sistema Único de Saúde(SUS) mediante articulação das escolas públicas, unidades básicas de saúde e unidades de Saúde da Família ${ }^{6}$.

O PSE objetiva contribuir para o enfrentamento das vulnerabilidades que comprometem o pleno desenvolvimento de crianças, adolescentes e jovens brasileiros. Essa iniciativa reconhece e acolhe as ações de integração entre saúde e educação já existentes e que têm impactado positivamente a qualidade de vida dos educandos ${ }^{6}$.

Dessa forma, o PSE constitui uma possibilidade de complemento de uma necessidade há tempos discutida: o fortalecimento da integração entre os setores educação e saúde, promovendo a intersetorialidade promulgada pelo SUS e a corresponsabilização entre estes setores, habituados a trabalhar isoladamente ${ }^{7}$.

A relação entre os serviços de saúde e as escolas representa um foco onde têm sido identificadas fragilidades,embora, cada vez mais, se considere fundamental um trabalho articulado entre ambas as partes para o sucesso da implementação de ações de educação para a saúde. O desenho do PSE parte justamente dessa necessidade de articular e integrar os setores da saúde e da educação para ampliar o alcance e o impacto das ações de saúde aos estudantes e seus familiares, por meio de ações intersetoriais. No PSE, a proposta é que essa articulação ocorra, principalmente, entre as Equipes de Saúde da Família (ESF) e as escolas do território de adscrição dessas equipes, atendendo às diversas demandas da comunidade escolar, na perspectiva da atenção integral e considerando o conceito positivo de saúde apresentado desde a Carta de Ottawa (1986) e reafirmado na Constituição Federal Brasileira de $1988^{8}$.

Historicamente, políticas de educação e saúde se estruturaram para que o seu funcionamento ocorresse de forma fragmentada, setorialmente. Isto contradiz o paradigma promocional da saúde, que expõe a necessidade de que o processo de produção do conhecimento e das políticas públicas ocorra por meio de construção e gestão compartilhadas, ou seja, por meio de ações intersetoriais em que haja um grau de abertura em cada setor para dialogar, estabelecendo corresponsabilidade e cogestão pela melhoria da qualidade de vida da população ${ }^{9}$.

Dessa maneira, unir a intersetorialidade - declarada como uma das diretrizes da Política Nacional de Promoção da Saúde ereafirmada pela Política Nacional de Atenção Básica (Pnab) como essencial para o desenvolvimento das ações de saúde ao rico e proveitoso território escolar é um propósito benéfico de produção de saúde ${ }^{10}$.

Considerando a complexidade dos programas de PS, que requerem abordagens amplas e diferenciadas tanto no desenvolvimento de suas ações quanto em seu processo analítico, e na tentativa de esclarecer as características do funcionamento de um programa intersetorial por meio da cooperação entre os diferentes setores e atores, este estudo de caso visa compreender como se dá a intersetorialidade no PSEsob a perspectiva da saúde e da educação no município de Olinda (PE). 


\section{PROCEDIMENTOS METODOLÓGICOS}

A presente pesquisa é resultado de uma dissertação de mestrado. Elegeu-se Olinda, pertencente à região metropolitana do Recife, no Estado de Pernambuco, como campo de pesquisa. De caráter qualitativo,a pesquisa utilizou a estratégia de estudo de caso, que possibilitou problematizar os limites e potencialidades identificados no processo de construção da intersetorialidade a partir da compreensão do PSE.

Elegeram-se as 20 primeiras escolas que aderiram ao PSE desde seu lançamento no município e que receberam a capacitação por meio de oficinas do programa. Os sujeitos desta pesquisa foram os agentes comunitários de saúde (ACS) e os professores comunitários, totalizando 19 entrevistados de ambos os setores, sendo esse número total determinado pelo efeito de saturação das informaç̃oes prestadas.

O levantamento dos dados constituiu-sepor duas entrevistas semiestruturadas. $\mathrm{O}$ roteiro da entrevista deste estudo abordou dois eixos temáticos correlacionáveis: Programa de Saúdena Escola e intersetorialidade.Utilizaram-se também diários de campo para o registro das situações observadas e/ou experimentadas durante o desenvolvimento da atividade de pesquisa. Os dados levantados foram organizados no software $\mathrm{NVivo}^{\circledast}{ }^{\circledR}$ versão 7.0 e analisados com o auxílio da Análise de Conteúdo proposta por Bardin ${ }^{11}$, seguindo as etapas de pré-análise, exploração do material e tratamento dos dados (inferência e interpretação). Após análise, a pesquisa foi dividida em duas categorias e respectivas subcategorias.

Atendidas as prerrogativas da Resolução nº 466/2012, do Conselho Nacional de Saúde para Pesquisa Científica em Seres Humanos,a proposta foi aprovada pelo Comitê de Ética em Pesquisa (CEP) da Universidade Federal de Pernambuco.

\section{RESULTADOS E DISCUSSÃO}

A presente pesquisa apresenta um diálogo significativo dos participantes com base em experiências e conhecimentos produzidos pelos profissionais de saúde e de educação que atuavam diretamente no PSE, possibilitando maior compreensão dos aspectos abordados. As apresentações serão feitas por categorias e subcategorias que foram corroboradas para essa sistematização.

Os profissionais, formados por cinco professores comunitários e 14 agentes comunitários de saúde, apresentaram idade média de 41 anos, e mais da metade deles trabalhava no PSE havia mais de três anos.

\section{Saúde na escola: uma realidade a ser ajustada}

Esta categoria foi constituída com o objetivo de compreender o Programa de Saúde na Escola em Olinda e sua importância e credibilidade enquanto política pública. Com base na análise das respostas, destacou-se o surgimento de subcategorias, que foram elencadas (Quadro 1) e discutidas segundo suas particularidades.

\section{QuAdro 1}

\begin{tabular}{|c|c|}
\hline \multicolumn{2}{|c|}{$\begin{array}{c}\text { QUADRO } 1 \\
\text { Saúde na escola: uma realidade a ser ajustada }\end{array}$} \\
\hline Subcategoria & Discurso \\
\hline $\begin{array}{l}\text { Conceito do PSE } \\
\text { alinhado ao conceito } \\
\text { governamental }\end{array}$ & $\begin{array}{l}\text { Eu entendo que é uma integração entre } \\
\text { a escola e os profissionais de saúde no } \\
\text { sentido de promover a saúde dentro } \\
\text { da escola, ou seja, um contato a mais, } \\
\text { uma melhoria na qualidade de vida dos } \\
\text { alunos, como também dos pais desses } \\
\text { alunos e dos professores porque, quando } \\
\text { fazemos essa parceria, quando fazemos } \\
\text { esse atendimento, ele não engloba só } \\
\text { os alunos, envolve também todos os } \\
\text { profissionais dentro da escola e seus } \\
\text { familiares. (Entrevistado H) }\end{array}$ \\
\hline $\begin{array}{l}\text { Importância } \\
\text { incontestável do } \\
\text { Programa de Saúde na } \\
\text { Escola }\end{array}$ & $\begin{array}{l}\text { Com certeza, o programa é importante. } \\
\text { O programa é maravilhoso, as ideias são } \\
\text { maravilhosas. (Entrevistado E) }\end{array}$ \\
\hline $\begin{array}{l}\text { Baixa credibilidade } \\
\text { do PSE }\end{array}$ & $\begin{array}{l}\text { Pra que o PSE, se não está tudo } \\
\text { amarrado, se realmente não leva ao pé da } \\
\text { letra? [...] Então é isso que tenho a dizer } \\
\text { do PSE, é um projeto maravilhoso, mas } \\
\text { tem defeito. (Entrevistado B) }\end{array}$ \\
\hline $\begin{array}{l}\text { Baixa credibilidade da } \\
\text { gestão municipal }\end{array}$ & $\begin{array}{l}\text { Não sei se é vontade política ou de } \\
\text { gestão, mas é por época, dependendo } \\
\text { da época política a gente tem mais } \\
\text { assistência. (Entrevistado G) }\end{array}$ \\
\hline $\begin{array}{l}\text { Deficiência de } \\
\text { capacitações }\end{array}$ & $\begin{array}{l}\text { E ainda existe uma dificuldade porque } \\
\text { eu fui treinado para fazer o Olhar Brasil } \\
\text { em uma hora e não me sinto seguro para } \\
\text { isso. (Entrevistado Q) }\end{array}$ \\
\hline
\end{tabular}

\section{Conceito do PSE alinhado ao conceito governamental}

No desvelar das opiniões dos profissionais acerca do conceito do Programa de Saúde na Escola, foi evidente a proximidade da compreensão do PSE pelos entrevistados de acordo com a literatura nacional dos Cadernos de Saúde e portarias dos ministérios da Educação e Saúde.

A Secretaria de Atenção à Saúde ${ }^{12}$ conceitua o Programa de Saúde na Escola como uma proposta de integração entre os ministérios da Saúde e Educação, na perspectiva de ampliar as ações específicas aos alunos da rede pública, solicitando dessa maneira uma força de trabalho composta por profissionais de ambos os setores e o reconhecimento do potencial da ESF, a partir da inserção de equipes de saúde na implementação do programa. 
Importância incontestável do Programa de Saúde na Escola É notável a compreensão da importância do programa pelos profissionais, pois, ao serem indagados sobre a importância do PSE, $100 \%$ dos entrevistados afirmaram que o programa era importante. Tambémressaltaram que a inclusão da saúde na escola traz benefícios para a qualidade de vida dos educandos e, consequentemente, melhora o acesso dessa população aos serviços de saúde, o que interfere positivamente na educação.

Essa relevância é confirmada por Gomes ${ }^{8}$, que destaca a escola como um ambiente de importantes interações sociais entre professores, alunos, familiares e profissionais de saúde, e onde surgem as diversas demandas e necessidades que podem ser problematizadas em seu contexto mais amplo.

A escola tem a potencialidade de atingir um grande número de indivíduos e, dado seu caráter educativo, ser efetiva na mudança de comportamentos e hábitos. Por tal motivo, os temas relacionados à saúde humana tradicionalmente fazem parte do cotidiano escolar no Brasil desde os primeiros anos de escolarização, ocupando em alguns momentos grande parte da carga horária de diversas disciplinas e mobilizando diversos atores na escola.

\section{Baixa credibilidade do PSE}

Observou-se que, ao mesmo tempo em que relatavam a importância do PSE, os entrevistados discorriam sobre a baixa credibilidade quanto à execução do programa. A credibilidade no PSE enquanto uma proposta que apoia a reorganização dos serviços de saúde, na perspectiva da intersetorialidade e do acolhimento da população juvenil, estava desacreditada, pois algumas diretrizes não eram seguidas corretamente.

Um dos pontos mais citados para o descrédito foi o Projeto Olhar Brasil, que visa identificar os problemas visuais relacionados à refração em alunos matriculados na rede pública. Observava-se uma fala fortemente indignada dos profissionais diante da execução incompleta e ineficaz do projeto. É de suma importância que haja aproximação entre o que é escrito e o que é desempenhado.

\section{Baixa credibilidade da gestão municipal}

De modo geral, as informações obtidas neste estudo indicaram que no nível local a participação da gestão no planejamento, monitoramento, implementação e avaliação não foi mencionada de forma significativa pelos profissionais. Isto entra em total desacordo com o modelo de gestão proposto pelo PSE, segundo o qual a gestão deve ser compartilhada de forma que tanto o planejamento quanto a execução das ações sejam realizados coletivamente, de forma a atender às necessidades $\mathrm{e}$ demandas locais.
Os gestores podem ser considerados peças fundamentais para coordenar ações intersetoriais, já que em nível local é possível unir os atores sociais em torno de problemas de sua realidade e buscar soluções próprias, direcionando o esforço conjunto para ações que influenciem a qualidade de vida da população, o que, de acordo com os entrevistados, não é encontrado.

É necessário o comprometimento de tais atores para que haja um reconhecimento e fortalecimento das estratégias de territorialização e corresponsabilização no campo saúde e educação, para a sustentabilidade do PSE como política.

\section{Deficiência de capacitações}

Entre as áreas temáticas de ação do PSE, encontra-se a proposta de educação permanente e capacitação de profissionais da saúde e da educação, com o objetivo de facilitar a comunicação e compreensão dos profissionais de ambos os setores de modo a fortalecer vínculos.

Há uma insatisfação geral dos profissionais, que relatam ter participado de apenas uma capacitação durante a implantação do programa. Quase todos passaram por apenas uma capacitação no início do programa, o que não se repetiu após quase cinco anos. Os professores indagaram mais, pois são responsáveis por ações de saúde para as quais não são amplamente qualificados, como, por exemplo, a detecção inicial de hanseníase e o teste de visão do Projeto Olhar Brasil nos alunos. Dessa forma, podemos concluir que as capacitações não foram suficientes para que os profissionais se preparassem adequadamente. Por ser o PSE um desenho inovador, é natural a necessidade de novas e contínuas capacitações.

\section{Intersetorialidade saúde/educação no PSE}

Nesta segunda etapa da análise das entrevistas, objetivamos compreender a intersetorialidade e o processo da intersetorialidade no PSE Olinda.

Assim, com base na análise dos discursos sobre esses questionamentos, destacamos o surgimento das subcategorias apresentadasno Quadro 2.

\section{Conceito de intersetorialidade restrito}

Cabe aqui ressaltar a dificuldade encontrada por alguns entrevistados em relação ao conceito de intersetorialidade.Embora todos os profissionais da educação soubessem responder à pergunta adequadamente, entre os ACS não havia clareza a respeito do tema. E ainda houve predominância do conceito vinculado apenas aos setores de saúde e educação.Com base nessa descrição, notamos a necessidade de estabelecer uma conceituação mais ampla em relação à intersetorialidade, des- 
viando a ideia de ser unicamente entre os setores de saúde e educação, no intuito de fortalecer esse processo.

\section{QuADro 2}

Intersetorialidade saúde/educação no PSE

\begin{tabular}{|l|l|}
\hline \multicolumn{1}{|c|}{ Subcategoria } & \multicolumn{1}{c|}{ Discurso } \\
\hline $\begin{array}{l}\text { Conceito de intersetorialidade } \\
\text { restrito }\end{array}$ & $\begin{array}{l}\text { É uma junção, uma coisa } \\
\text { puxa a outra, eu acho que se } \\
\text { não fosse dessa forma não } \\
\text { daria certo. Essa junção ajuda. } \\
\text { (Entrevistado R) }\end{array}$ \\
\hline
\end{tabular}

Existência da intersetorialidade saúde/educação no PSE

Conflito de interesses entre os setores saúde e educação

Deficiência na comunicação entre os setores

Poucos interlocutores além da saúde e educação
Existe sim, os dois setores unidos em prol dos alunos. (Entrevistado I)

Minha função era interagir, abrir espaço, organizar, planejar, como eu irei fazer com que vocês entrem na sala de aula; porque não é assim, chegar e pronto, existem professoras, existe planejamento, existem horários, e não estar preenchendo ficha de aluno e cadastro de aluno e não estar fazendo exame de aluno, eu não me formei para isso... (Entrevistado E)

Às vezes, eles dizem que vai haver uma ação na escola, quando a gente chega no dia a escola não está sabendo ou então a escola está sabendo e a gente não está. Aí às vezes só isso é que complica. (Entrevistado C)

Temos ajuda da Secretaria do Esporte e Cultura, mas apenas quando pedimos. (Entrevistado P)

A intersetorialidade surge como um caminho para a estruturação de ações coletivas mais complexas, que deem conta da realidade e de suas diversas variantes. Espera-se encontrar ações conjuntas que persigam o mesmo objetivo entre setores diferentes, considerando a complexidade do setor saúde e a necessidade de abordagens mais amplas para o enfrentamento dos problemas que afetam a saúde da população ${ }^{13 .}$

\section{Existência da intersetorialidade saúde/educação no PSE}

Os profissionais reconheciam a existência da prática da intersetorialidade no desenvolvimento do Programa Saúde na Escola. Afirmaram que, para conduzir um programa como esse,

seria preciso trabalhar intersetorialmente para que as ações não se configurassem apenas como ações específicas da saúde ou da educação, mas possibilitassem formar um espaço integrado, participativo e em transformação, sob a perspectiva da PS. Apenas três respondentes relataram não haver intersetorialidade entre saúde e educação em seu território.

Assim, como Westphal e Mendes ${ }^{14}$, entendemos que, embora com dificuldade, a intersetorialidade é uma forma de gestão possível e muito eficiente em termos da promoção do desenvolvimento econômico e social.

\section{Conflito de interesse entre os setores saúde e educação}

Verificamos no depoimento de alguns entrevistados a existência de um conflito de interesses entre os setores da saúde e educação. A crítica dos atores a esse fator se dá em função de cada setor possuir suas agendas, assim como várias atribuições predefinidas, provocando sobrecarga e induzindo, muitas vezes, ao conflito de interesses entre os setores.

De acordo com Monteiro e Bizzo ${ }^{15}$, a entrada de profissionais da saúde no ambiente escolar pode acabar por desprestigiar a ação e o discurso dos profissionais da educação, mais especificamente do professor, em assuntos relativos ao tema - se é preciso convidar profissionais externos para desenvolver atividades específicas, há o entendimento tácito de que os docentes não são capazes de ministrar tais conteúdos sem esse auxílio de especialista.

Diante do exposto, caberia pensar em estratégias para controlar tais dificuldades na governabilidade dos profissionais, uma vez que, de certa forma, podem influenciar a mobilização e o desenvolvimento de competências em parceria.

A parceria entre educação e saúde precisa representar um espaço de negociação e solidariedade no enfrentamento de divergências e conflitos internos em cada um dos setores que estejam vinculados a modelos de atenção e paradigmas de base biomédica. Sendo assim, a intersetorialidade no campo da saúde na escola constitui uma estratégia para a concretização da PS na área.

\section{Deficiência na comunicação entre os setores}

É sabido que o diálogo entre os setores saúde e educação para a formulação, implantação, acompanhamento ou avaliação conjunta de políticas e programas de PS no cenário da escola ainda se apresenta como um grande desafio para o PSE, o quefoi ratificado pela fala dos profissionais entrevistados.

O obstáculo na comunicação entre os setores foi um grande entrave para a concretização da intersetorialidade percebido durante a pesquisa.Osobstáculos alegados pelos profissionais foram a falta de tempo e a sobrecarga de trabalho, que 
podem ser entendidas como fatores coadjuvantes na ausência de envolvimento total. Nessa direção, caberia pensar em estratégias para incrementar esse diálogo, que acreditamos ser um ponto-chave para possibilitar o refletir-agir e potencializar as ações intersetoriais.

\section{Poucos interlocutores além da saúde e educação}

Em relação aos outros setores governamentais que se envolvem intersetorialmente nas ações que o PSE desenvolve no município de Olinda, além da saúde e educação, foram citados apenas a Secretaria do Esporte e da Cultura e a Secretaria de Ação Social e setores não governamentais, como o Banco Santander e igrejas locais. Não se percebe a existência de uma rede de apoio formal ao programa. Desta forma, estão envolvidos diretamente apenas os setores da educação (a escola) e da saúde (Unidades de Atenção Primária em Saúde), o que dificulta contemplar todas as necessidades dos educandos.

Observou-se que não havia muitas outras parcerias além da saúde e educação. Ademais, outras parcerias eram obtidas apenas quando necessário. É indispensável uma ação mais articulada e sistemática entre os serviços de saúde e educação e as demais redes sociais, que já existem e se integram cotidianamente, sem, contudo, se darem conta disso. A estruturação dessas redes possibilitará o fortalecimento e a reorientação das práticas, dos saberes e das lutas, dinamizando e potencializando ações de saúde e educação no PSE. O PSE deve também se articular localmente com outros projetos e programas voltados à atenção escolar para reforçar as iniciativas existentes.

\section{CONSIDERAÇÕES FINAIS}

A implantação do PSE permitiu aos profissionais de saúde a percepção do seu papel social de educador e possibilitou aos adolescentes maior contato com a equipe da ESF. Consideramos que a aproximação entre escola e unidade de saúde contribuiu para ajudar os adolescentes a transformar a informação científica em comportamentos saudáveis. Compreendemos,com base na experiência vivenciada, que diversificar os locais de atendimento à saúde, bem como colocar em prática estratégias educativas que vão além das meras palestras tradicionais ampliam as possibilidades de melhoria da assistência prestada pela equipe da ESF, além de dar maior visibilidade às atividades desenvolvidas na atenção primária.

Nas entrevistas, percebe-se que há uma tentativa de intersetorialidade entre saúde e educação, o que não perpassa outros setores. No entanto, essa tentativa é repleta de entraves alheios às possibilidades dos setores. Sendo assim, a intersetorialidade foi considerada uma possibilidade de ação governamental para superar a fragmentação dos serviços, influenciando o planejamento, a execução e a avaliação de resultados alcançados. Identificada como um processo difícil e não espontâneo, exige habilidades para lidar com conflitos, contradições e divergências.

Percebe-se neste estudo que a sustentabilidade na realização de práticas intersetoriais é ainda um desafio a ser conquistado, mas é uma prática possível, principalmente quando amparada e estimulada por uma boa gestão local. O conceito e a prática da intersetorialidade precisam ser inseridos na rotina de gestores e profissionais de saúde e educação para que ações de promoção de saúde não se tornem a repetição de modelos conhecidos e reconhecidamente pouco impactantes na melhoria de condições de saúde da população. A responsabilidade sobre essa nova forma de olhar e cuidar não deve ser atribuída exclusivamente a esses atores, mas em políticas voltadas a facilitar a articulação dos setores governamentais e não governamentais, controle e responsabilização social, estimulando a educação continuada de profissionais de saúde e educação no protagonismo dessas ações.

O impacto em saúde das ações intersetoriais desenvolvidas pelas equipes não foi o foco deste estudo, mas a avaliação qualitativa mostrou um rompimento de ações unicamente centradas na atenção às doenças. As respostas e discursos dos profissionais demonstraram que é possível, quando há conhecimento e boa vontade, mudar e melhorar as realidades locais.

Uma diferença substancial desta pesquisa foi o fato de os profissionais serem representados por profissionais de nível superior (professores) e de nível médio (agentes comunitários de saúde), escolhidos por atuarem diretamente no PSE.

Entre as dificuldades encontradas neste estudo, destacamos a escassez de estudos recentes e semelhantes sobre o PSE.

O Programa Saúde na Escola, embora apresente algumas dificuldades estruturais e gerenciais no âmbito dos escolares da rede municipal de ensino de Olinda, evidencia-se como ferramenta capaz de propiciar melhorias na qualidade de vida dos alunos que compõem as escolas, pois desenvolve ações benéficas e eficazes relacionadas à saúde e educação.

O dilema passa a ser operar uma política necessariamente intersetorial explicitada num ambiente com atores que possuem agendas setoriais previamente montadas e sem espaço suficiente para a correta execução da política, o que leva à dificuldade de conciliar os tempos institucionais dos vários setores e o comprometimento e envolvimento setorial, impedindo a sustentabilidade das ações. Mudanças estão sendo implementadas, mas o que se nota é a escassez de capacitação e educação permanente (como preconizado pelo programa), além da inexistência de protocolos que norteiem o desenvolvimento de ações intersetoriais. 
Nosso estudo se propôs a analisar os conhecimentos e práticas da intersetorialidade no PSE após alguns anos de sua implantação. Observou-se que a intersetorialidade já é uma prática conhecida entre a saúde e a educação, além de ser conhecida como um pilar do PSE, porém ainda não é tão estimulada em razão de entraves do cotidiano dos profissionais. Há uma tentativa de consolidação de práticas intersetoriais, que, na maioria das vezes,são substituídas por ações pontuais. De posse desses dados, podemos pensar em intervenções mais concretas para fortalecer a intersetorialidade e as ações intersetoriais no PSE.

\section{REFERÊNCIAS}

1. Silva KL. Promoção da Saúde em espaços sociais da vida cotidiana. Belo Horizonte; 2009. Doutorado [Tese] - Escola de Enfermagem da UFMG.

2. Wimmer GF, Figueredo GO. Ação coletiva para qualidade de vida: autonomia, transdisciplinaridade e intersetorialidade. Ciên Saúde Coletiva; 2006. Disponível em: <http:/ / www.scielo.br/scielo.php?pid=S003471672010000500011 \&script=sci_arttext $>$. Acesso em: 29 abr. 2012.

3. Westphal MF. Promoção da Saúde e a qualidade de vida. 2007. In: Fernandez JCA; Mendes R (Org). Promoção da Saúde e Gestão Local. Disponível em: <http://cmdss2011. org/site/wp-content/uploads/2011/07/promocao-da-saude-e-gestao-local.pdf $>$. Acesso em: 30 out. 2012.

4. Teixera CF, Paim JS. Planejamento e programação de ações intersetoriais para a promoção da saúde e da qualidade de vida. Revista de Administração Pública. FGV. Rio de Janeiro; 2000.

5. Brasil. Ministério da Saúde. Secretaria de Ciência, Tecnologia e Insumos Estratégicos. Departamento de Ciência e Tecnologia. Agenda nacional de prioridades de pesquisa em saúde. 2ª edição. Brasília: MS; 2008.

6. Brasil. Ministério da Saúde. Secretaria de Atenção à Saúde. Departamento de Atenção Básica. Passo a passo PSE: tecendo caminhos da intersetorialidade. Brasília: MS; 2011.

7. Santiago L, Rodrigues MTP, Oliveira Junior AD, Moreira TMM. Implantação do Programa Saúde na escola em Fortaleza-CE: atuação de equipe da Estratégia Saúde da Família. Rev. bras. Enferm, Brasília; 2012. Disponível em: http:/ / www.scielo.br/scielo.php?script=sci_arttext\&pid =S0034-71672012000600020. Acesso em: 23 jan. 2014.

8. Gomes LC. O desafio da intersetorialidade: a experiência do Programa Saúde na Escola (PSE) em Manguinhos, no Município do Rio de Janeiro. Rio de Janeiro; 2012. Mestrado [Dissertação] - Escola Nacional de Saúde Pública Sergio Arouca.
9. Ferreira IRC, Vosgerau DSR, Moysés SJ, Moysés ST. Diplomas Normativos do Programa Saúde na Escola: análise de conteúdo associada à ferramenta ATLAS TI. Ciênc. saúde coletiva, Rio de Janeiro; 2012. Disponível em: <http:// www.scielosp.org/pdf/csc/v17n12/23.pdf >. Acesso em: 05 jan. 2013.

10. Brasil. Ministério da Saúde. Secretaria de Vigilância em Saúde. Secretaria de Atenção à Saúde. Política Nacional de Promoção da Saúde. Brasília: MS; 2006.

11. Bardin L. Análise de Conteúdo. $4^{a}$ ed. Lisboa: Edições 70; 2009.

12. Brasil. Ministério da Saúde. Secretaria de Atenção à Saúde. Departamento de Atenção Básica. Saúde na Escola. Brasília: MS; 2009.

13. Junqueira LP. A gestão intersetorial das políticas sociais e o terceiro setor. Saude e Socied 2004; 13(1):25-36.

14. Westphal MF, Mendes R. Cidade saudável: uma experiência de interdisciplinaridade e intersetorialidade. Revista de Administração Pública; 2000.

15. Monteiro PHN, Bizzo N. A saúde na escola: análise dos documentos de referência nos quarenta anos de obrigatoriedade dos programas de saúde, 1971-2011. História, Ciências, Saúde - Manguinhos; 2015.

\section{CONTRIBUIÇÃO DOS AUTORES}

Isabelle Carolline Veríssimo de Farias participou da concepção, revisão bibliográfica, pesquisa, metodologia, análise das entrevistas, interpretação dos dados, construção da dissertação e redação final. Ronice Maria Pereira Franco de Sá participou de todas as etapas, orientando o trabalho e a redação final. Nilcema Figueiredo e Abel Menezes Filho participaram como examinadores da revisão da pesquisa durante sua execução.

\section{CONFLITO DE INTERESSES}

Os autores declararam que dentro dos últimos 5 anos e para o futuro próximo que não possuíam conflito de interesse de ordem: pessoal, comercial, acadêmico, político e financeiro no manuscrito. Declararam também que todo apoio financeiro e material recebido para o desenvolvimento da pesquisa que resultou na elaboração do manuscrito foram financiadas pela autora principal, não houve financiamentos externos.

\section{ENDEREÇO PARA CORRESPONDÊNCIA}

Isabelle Carolline Verissimo de Farias

Rua General Artur Óscar, 30. Studio Rosarinho Prince.

Encruzilhada - Recife

CEP: 52050-430

E-mail: belleverissimo@hotmail.com 